\title{
Assistência de enfermagem na qualidade de vida das pacientes pós mastectomizadas: revisão de literatura
}

\author{
Nursing assistance in the quality of life of patients after mastectomized: literature review \\ La atención de enfermería en la calidad de vida del paciente post mastectomizado: revisión de la \\ literatura
}

Recebido: 26/09/2021 | Revisado: 02/10/2021 | Aceito: 06/10/2021 | Publicado: 10/10/2021

\author{
Maiara Rodrigues Maia \\ ORCID: https://orcid.org/0000-0002-6719-2911 \\ Hospital Sírio Libanês, Brasil \\ E-mail: maiara.enfermagem@ hotmail.com \\ Lorena Esmeralda Nascimento Celeste \\ ORCID: https://orcid.org/0000-0001-8217-620X \\ Universidade Salvador, Brasil \\ E-mail: lory.celeste@yahoo.com.br \\ Marks Passos Santos \\ ORCID: https://orcid.org/0000-0003-1180-404X \\ Faculdade Ages de Jacobina, Brasil \\ E-mail: enfer.marks@ @otmail.com \\ Emanuel Osvaldo de Sousa \\ ORCID: https://orcid.org/0000-0003-2825-4275 \\ Centro Universitário Facid, Brasil \\ E-mail: emanfisio@hotmail.com \\ Lucília da Costa Silva \\ ORCID: https://orcid.org/0000-0001-9386-5684 \\ Centro Universitário Santo Agostinho, Brasil \\ E-mail: luciliafisio@outlook.com \\ Alex de Souza Silva \\ ORCID: https://orcid.org/0000-0001-8136-9659 \\ Universidade Católica de Goiás, Brasil \\ E-mail: alexss21silva@gmail.com \\ Héverson Batista Ferreira \\ ORCID: https://orcid.org/0000-0002-0697-6982 \\ Universidade Potiguar, Brasil \\ E-mail: eversonbatst@gmail.com \\ Victor Guilherme Pereira da Silva Marques \\ ORCID: https://orcid.org/0000-0002-7441-9811 \\ Centro Universitário do Piauí, Brasil \\ E-mail: guilhermevictor521@gmail.com \\ Diego Bruno Brito Cerqueira \\ ORCID: https://orcid.org/0000-0002-8083-1634 \\ Faculdade Integral Diferencial, Brasil \\ E-mail: diegocerqueirafisioterapeuta@ hotmail.com
}

\begin{abstract}
Resumo
A neoplasia mamária, e a superioridade na incidência e, sobretudo, pelos seus efeitos psicossociais, torna-se o mais temeroso no sexo feminino. Objetivo: Realizar uma revisão de literatura sobre o cuidado de enfermagem nas mulheres pós mastectomia. Metodologia: Estudo de revisão bibliográfica narrativa selecionou-se 101 artigos e utilizamos 22, para o desenvolvimento do estudo no período entre outubro de 2018 e maio de 2019. Resultados: A equipe multidisciplinar, onde abrangem uma diversidade de profissionais, voltando-se aos aspectos de humanização do atendimento. Os aspectos emocionais da paciente e família devem ser vistos de forma especial, onde à unidade oncológica é um ambiente estressante e desgastante no qual o apoio da equipe de enfermagem é essencial para amenizar os conflitos existentes. Conclusão: Entretanto observou-se fundamental importância no envolvimento da unidade de saúde, como um todo nos aspectos materiais e pessoal, no acolhimento e na educação continuada, na forma de condução das pacientes em acontecimentos ou incidentes inesperados, que são corriqueiros nos ambientes de cuidado, e atendimento ao paciente oncológico, considerando-se necessário que exista a humanização do cuidado.
\end{abstract}

Palavras-chave: Mastectomia; Qualidade de vida; Assistência de enfermagem. 


\begin{abstract}
Breast neoplasm, and the superiority in incidence and, above all, for its psychosocial effects, becomes the most fearful in the female sex. Objetivo: Realizar uma revisão de literatura sobre o cuidado de enfermagem nas mulheres pós mastectomia. Methodology: Narrative literature review study selected 101 articles and we used 22, for the development of the study in the period between October 2018 and May 2019. Results: The multidisciplinary team, where they encompass a diversity of professionals, turning to the aspects of humanization of care. The emotional aspects of the patient and family should be seen in a special way, where the oncology unit is a stressful and exhausting environment in which the support of the nursing team is essential to ease the existing conflicts. Conclusion: However, it was observed the fundamental importance of the health care unit's involvement, as a whole, in material and personal aspects, in the reception and continuing education, in the way patients are conducted in unexpected events or incidents, which are common in care environments, and in the care of oncologic patients, considering it necessary to humanize care.
\end{abstract}

Keywords: Mastectomy; Quality of life; Nursing care.

\title{
Resumen
}

La neoplasia de mama, y la superioridad en la incidencia y, sobre todo, por sus efectos psicosociales, se convierte en la más temida en el sexo femenino. Objetivo: Realizar una revisión de la literatura sobre el cuidado de la salud de las mujeres sometidas a mastectomía. Metodología: El estudio de revisión bibliográfica narrativa seleccionó 101 artículos y utilizamos 22, para el desarrollo del estudio en el periodo comprendido entre octubre de 2018 y mayo de 2019. Resultados: El equipo multidisciplinar, en el que se engloban una diversidad de profesionales, volcándose en los aspectos de humanización de la atención. Los aspectos emocionales del paciente y la familia deben ser vistos de manera especial, donde la unidad de oncología es un ambiente estresante y agotador en el que el apoyo del equipo de enfermería es esencial para aliviar los conflictos existentes. Conclusión: Por lo tanto, se observa la importancia fundamental en el envolvimiento de la unidad de salud, como un todo en los aspectos materiales y personales, en el acolchado y en la educación continuada, en la forma de conducción de los pacientes en acontecimientos os incidentes inesperados, que se corren en los ambientes de cuidado, y en la atención al paciente oncológico, considerando necesario que exista la humanización del cuidado.

Palabras clave: Mastectomía; Calidad de vida; Cuidado de enfermera.

\section{Introdução}

O câncer de mama, devido à sua alta incidência e, sobretudo, pelos seus efeitos psicossociais, é considerado o mais temido entre as mulheres. A nível mundial, o câncer de mama, em relação as neoplasias malignas, apresenta-se como o segundo tipo de câncer com maior freqüência e o primeiro entre o sexo feminino. Esse tipo de câncer manifesta-se perante a forma de nódulos e, geralmente, podem ser detectados pelas próprias mulheres, mediante a realização do autoexame (Duarte \& Andrade, 2003 apud Bertolo \& Pauli, 2008).

A neoplasia maligna da mama é o câncer mais incidente e a segunda causa de óbito mundialmente entre as mulheres. No Brasil, os últimos dados tiveram como estimativa no período de 2018 um percentual de 29,5\% de novas ocorrências, que indicam um número de 59.700, de neoplasia maligna da mama feminina detectados. Apesar de ser apontado, na maior parte dos casos, um câncer com bom prognóstico, caso seja detectado e tratado precocemente, a neoplasia maligna da mama ainda está associada a uma alta taxa de mortalidade neste país (Instituto Nacional de Câncer [INCA], 2018).

As modalidades terapêuticas incluem quimioterapia, radioterapia, cirurgia e hormonioterapia. A mastectomia se caracteriza pela remoção total da mama, dependendo da necessidade e comprometimento. As limitações funcionais e emocionais decorrentes da mastectomizada interferem na qualidade de vida das mulheres (Monteiro \& Paiva, 2018).

Segundo Lopes, Moura, Raso, Vedovato e Ribeiro (2013) a mastectomia parcial ou radical modificada, dentre outros tratamentos existentes, é a mais indicada para o câncer de mama. Essa modalidade sofreu grandes avanços nos últimos tempos devido à evolução científica, e a técnica cirúrgica de mastectomia com reconstrução mamária imediata pode ser apontada um exemplo deste avanço.

A mastectomia é um dos tratamentos prováveis para a maior parte das mulheres com câncer de mama. Ao submeter-se à retirada da mama ou parte dela, certamente, a mulher passará por uma grande mudança, vivenciando, assim, um comprometimento físico, emocional e social (Pereira, Rosenhein, Bulhosa, Lunard, \& Filho, 2006). 
A reconstrução mamária neste contexto, evidência uma viabilidade de reabilitação satisfatória atualmente para as mulheres que precisam se submeter à mastectomia radical, como o tratamento relevante para o câncer de mama. A reconstrução necessitará de vários aspectos: peso, altura, idade, tratamento pregresso ou complementar com radioterapia, condição de saúde e outras cirurgias realizadas. A evolução dos procedimentos de cirurgia plástica atualmente tem proporcionado resultados adequados para a viabilidade estética e psicológica da mulher, ao restringir o impacto causado pela mutilação (Duarte \& Andrade, 2003).

É bom lembrar que a mama não corresponde apenas ao aspecto físico, ela é vista como um símbolo de feminilidade, por isso é motivo de orgulho para a mulher e admiração para os homens, possuindo um significado afetivo e psicológico. Sendo assim, a palavra câncer traz um estigma muito forte para a mulher, pois além de estar associada à morte, pode afetar uma parte tão valorizada do seu corpo (Moura, Silva, Oliveira, \& Moura, 2010).

Segundo Ribeiro, Sousa, Andrade, Brito e Albuquerque (2014) os seios são uma parte do corpo que representa a identidade feminina da mulher. Mas, perante o cancro e a necessidade de se proceder a uma mastectomia, a dor física e principalmente a psicológica toma conta da mulher que vê perder uma parte de si e da sua identidade e essência enquanto mulher.

A qualidade de vida $(\mathrm{QV})$ pode ser definida como a percepção que um indivíduo tem de sua posição na vida, no contexto da cultura, sistemas de valores nos quais ele vive em relação aos seus objetivos, expectativas, padrões e preocupações (Almeida-Brasil et al., 2017).

A escolha desse, tema qualidade de vida das pacientes pós mastectomizadas, surgiu devido à importância da temática para a área acadêmica oncológica dentro do contexto da enfermagem, vindo contribuir com informações e dados relevantes nos índices de atendimento eficaz e acolhimento humanizado à pacientes na unidade de atendimento oncológico, e conscientização da importância da reconstrução mamária, no tempo adequado, para as pacientes pós mastectomizadas seguindo as normas corretas.

Uma vez que a enfermagem tem papel fundamental na unidade de atendimento oncológico, desenvolve função crucial na orientação, cuidados e acolhimento humanizado as pacientes em situação de pós cirurgia oncológica mamaria. Devido à importância do atendimento humanizado, o saber acolher do enfermeiro, nas aflições das mulheres em situação inicial de rejeição ao próprio corpo em detrimento ao avançado da patologia o presente estudo bibliográfico justifica-se diante do desenvolvimento da enfermagem, como fator preponderante na questão da temática Assistência de enfermagem na qualidade de vida das pacientes pós mastectomizadas, com relação aos cuidados psicológicos e emocionais, e a qualidade do atendimento para com as pacientes, no contexto da reconstrução mamária, junto à diminuição dos altos índices de mutilação mamária e sofrimento emocional das mulheres.

$\mathrm{Na}$ unidade oncológica, os altos índices de mulheres mutiladas podem estar atrelado à remoção do tumor, e com isso desencadeiam os problemas físicos e psicológicos, e essa resposta é individual. Cabe a enfermagem, junto ao serviço multidisciplinar saber respeitar e conduzir o processo de cuidado, e as formas de manejar a paciente de uma forma ágil e delicada, para minimizar o sofrimento dela. Entretanto complementa-se a importância do desenvolvimento do estudo através da temática pacientes pós mastectomizadas para a área acadêmica oncológica. Dentro do contexto da enfermagem, podemos analisar tão quanto essas informações, contribuem em caráter demonstrativo nos índices de atendimento eficaz e humanizado na unidade de atendimento oncológico, e a conscientização das pacientes, sobre a importância da reconstrução mamária, com indicações adequadas para a realização dela.

Portanto, a enfermagem tem grande parcela no processo de acolhimento, ao desempenhar função crucial na reestruturação da autoestima da mulher, ofertando a segurança adequada à mesma, transmitindo conforto e harmonia, desenvolvendo o seu papel fundamental nos atendimentos oncológicos. Torna-se um estudo interessante, e cientificamente 
abrangente, relatando dentre as diversas funções do enfermeiro no cuidado oncológico,com pacientes pós mastectomizadas junto ao atendimento humanizado, e a preservação da autoimagem dessas pacientes e com ressalva para o embasamento teórico a respeito do tema estabelecido.

\section{Metodologia}

Trata-se de um trabalho de revisão bibliográfica narrativa, destinada a avaliar a qualidade de vida das pacientes em situação de mastectomia, assim como identificar as dificuldades enfrentas pelas mesmas na inserção a vida social com relação aos aspectos psicológicos.

A revisão de literatura (ou revisão narrativa) é sempre recomendada para o levantamento da produção científica disponível e para a reconstrução de redes de pensamentos e conceitos, que articulam saberes de diversas fontes na tentativa de trilhar caminhos na direção daquilo que se deseja conhecer (Gomes \& Caminha, 2013).

Será uma pesquisa descritiva, do ponto de vista dos objetivos, segundo (Gil, 1994) a pesquisa do ponto dos procedimentos técnicos é bibliográfica (segundo Selltiz, Wrightsman, \& Cook, 1967, apud Gil, 2002) elaborada a partir de material já publicado, como livros, artigos, periódicos, Internet.

A estratégia de análise e seleção da amostra foi à pesquisa on-line das publicações científicas, através das seguintes bases de dados: Medical Literature Analysis and Retrieval System Online (MEDLINE), Base de Dados Bibliográficas Especializada na Área de Enfermagem (BDENF), Biblioteca Virtual em Saúde (BVS) e Web of Science. Foram utilizados os descritores "Mastectomia", "Qualidade de Vida" e "Assistência de Enfermagem" realizando-se a busca destes termos no título, resumo ou corpo do artigo, mediante aplicação do operador booleano AND.

Após este levantamento, será realizada uma primeira seleção a partir dos títulos, depois a leitura dos resumos e, após, procederemos à seleção e leitura na íntegra dos artigos a serem analisados. No qual foram selecionamos 101 artigos, sendo utilizados 22, para o desenvolvimento do estudo no período entre outubro de 2018 e Maio de 2019.

Como critérios de inclusão: estudos na íntegra, com publicação no período de 2001 a 2019, preferencialmente nos idiomas português e inglês. Estudos que abordassem os cuidados de enfermagem prestados as pacientes submetidas à mastectomização, com ênfase na qualidade de vida, estudos observacionais e apresentasse um contexto nos descritores apresentados: Câncer de mama; mastectomia e inserção social dessas mulheres nos espaços comum.

Como Critérios de exclusão: estudos repetidos entre as bases, os indisponíveis na íntegra e os que não abordassem o tema central deste estudo. Estudos de revisão de literatura, sem resumos, trabalhos de conclusão de curso e relatos de caso foram excluídos.

Além disso, também foi utilizado o fluxograma PRISMA para apresentar o processo de identificação, triagem e inclusão dos estudos. 
Figura 1 - Fluxograma do método de busca e triagem, Salvador 2021.

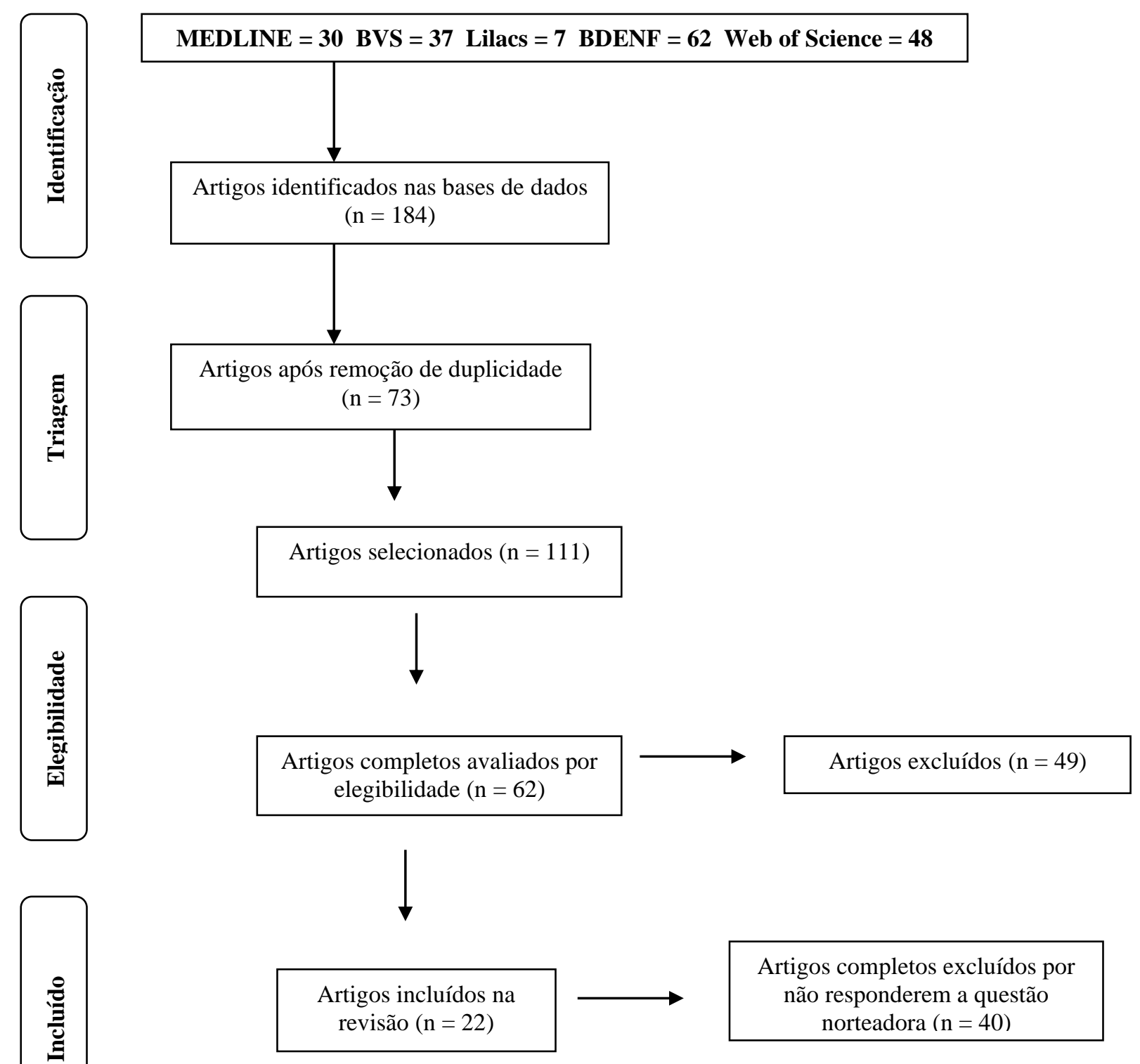

Fonte: Autores (2021).

\section{Resultados e Discussão}

O seio é o símbolo de feminilidade, e, dentro de uma espiral de complexidade, o seu acometimento expõe as pacientes a uma finitude de questões: o seu posicionamento como mulher, atraente e feminina, ou a mãe que amamenta. Portanto, entender a oncopsiquiatria das neoplasias mamárias é uma maneira de entender o universo feminino (Cantinelli et al., 2006).

Segundo Reis, Panobianco e Gradim (2019) ao deparar-se com um diagnostico agressivo como o carcinoma de mama, especialmente ao que se refere ao sexo frágil, é repleta de significados, visto que a patologia e seus tratamentos promovem uma mudança significativa na vida daquelas que o recebem, alterando seu corpo, o seu estado emocional e sua rotina de vida.

A palavra câncer é vista pela sociedade como uma fase irreversível cheio de significados, que representa uma grave e constante adversidade no contexto mundial de saúde. A denominação de câncer se estabelece a um conjunto de mais de 100 doenças que têm em comum o crescimento desordenado de células, que invadem tecidos e órgãos. Dividindo-se rapidamente, 
estas células tendem a ser muito agressivas e incontroláveis, determinando a formação de tumores, que podem espalhar-se para outras regiões do corpo (INCA, 2019 apud Moura et al., 2010).

A doença maligna das mamas, devido à sua alta incidência e, sobretudo, pelos seus efeitos psicossociais, é considerada o mais agressivo e impactante para o sexo feminino. Ao nível mundial, as doenças malignas mamárias representam em nível mundial e nacional, um dos mais agressivos e temidos, apresentando-se em alta escala mantendo-se como o mais temido entre o sexo feminino. Em sua grande maioria apresenta-se sob a forma de massa palpável, sendo observado pela própria mulher, por meio da prática do autoexame. O carcinoma mamário tem como base uma desordenação, por parte das multiplicações de células pré-determinadas que reproduzem-se em elevada velocidade, transformando e gerando o aparecimento de massas ou neoplasias malignas que podem vir a afetar os tecidos vizinhos e provocar metástases (Duarte \& Andrade, 2003 apud Bertolo \& Pauli, 2008).

A neoplasia maligna que afetam os seios é o câncer mais incidente e a segunda causa de óbito em mulheres mundialmente. Em nosso País, Brasil os últimos dados tiveram como estimativas em 2018 um percentual de 29,5\% de casos descobertos que indicam um número de 59.700, de neoplasia maligna da mama feminina detectados. Em sua grande abordagem, na maioria dos casos, é um câncer com bom prognóstico, caso tenha uma descoberta em fase inicial e tomada todas as medidas necessárias precocemente, a neoplasia maligna da mama ainda está associada a uma alta taxa de mortalidade neste país (INCA, 2018).

Segundo o Thuler (2003), a precaução primária objetiva diminuir a incidência de uma doença numa população, reduzindo o risco de surgimento de novas demandas, ao prevenir a exposição aos fatores que levam ao seu desenvolvimento, interromper seus efeitos ou alterar as respostas do hospedeiro a essa exposição, impedindo que ocorra seu início biológico.

A doença mamaria é relatada como de boa estatística se de maneira oportuna os cuidados iniciais forem efetivos, e o inicio ao manejo da doença sejam realizados oportunamente, sendo o principal fator que dificulta o tratamento é o avançado estado que a paciente encontra-se ao descobrir a doença. Em nosso país, a maioria dos casos é diagnosticada em estágios avançados (III e IV), correspondendo a uma demanda de $60 \%$ nos diagnósticos, por isso o elevado índice de mastectomias realizadas no Brasil é considerado alto (Silva, 2018).

Segundo Cabral, Giatti, Casale e Cherchiglia (2019) a demora no diagnóstico e no início do tratamento tem sido associada ao pior prognóstico da patologia e diminuição da sobrevida. Atrasos entre a suspeita e a primeira consulta com especialista são frequentemente associados a características da paciente: idade avançada, baixa escolaridade, informação não adequada sobre a doença, não ter convenio médico e falta de recursos financeiros na busca pelo profissional. Já atrasos nos intervalos entre a consulta, é a descoberta e o tratamento geralmente relacionam-se ao contexto assistencial-

O profissional enfermeiro que cuida da mulher precisa conhecer as várias opções de manejo de cuidado para discutilas com a paciente e estar ciente da informação repassada pelo médico a sua. Em função disso, a enfermeira exerce um papel de imensa importância na vida destas mulheres, pois é esta que identifica as preocupações, ansiedades e medos das pacientes, orientando-as e apoiando-as psicologicamente. Uma conduta cuidadosa e calma, estabelecendo um diálogo entre paciente e enfermeira, pode controlar a ansiedade da mulher frente ao diagnóstico e durante o tratamento (Bertolo \& Pauli, 2008).

Diante disto é notória a grande importância que a equipe de enfermagem oncológica, pode desempenhar no início do diagnóstico de câncer no acolhimento das mulheres ao receber a notícia. A enfermagem pode orientar e auxiliar a paciente no tratamento e nas condutas que devem ser tomadas pela equipe confortando e apoiando a mesma e sua família, no após receberem o diagnóstico de câncer. Prestando assim um atendimento diferencial e humanizado, para que haja dessa forma uma diminuição nos altos índices de abandono ao tratamento e de desassistência a paciente. 
Avaliar a paciente diagnosticada e submetida a tratamento por câncer de mama é questão importante e, muitas vezes, tarefa a cargo do oncologista, do cirurgião, da enfermeira ou dos demais membros da equipe multidisciplinar, já que raramente o psiquiatra ou o psicólogo entram em cena no início da abordagem (Cantinelli et al., 2006).

Os programas de rastreamento para câncer de mama têm como objetivo identificar mulheres que se encontram em estágio precoce da doença. Atualmente a mamografia (MMG), é o método preconizado para rastreamento na rotina da atenção integral à saúde da mulher. A mamografia é o único exame cuja aplicação em programas de rastreamento apresenta eficácia comprovada na redução da mortalidade do câncer de mama. (INCA, 2019 apud Thuler, 2003).

A mamografia é apontada como o principal método diagnóstico do câncer de mama em estágio inicial, capaz de detectar alterações ainda não palpáveis e favorecendo, assim, o tratamento precoce, mais efetivo, menos agressivo, com melhores resultados estéticos e eventos adversos reduzidos. Entretanto, embora vários estudos mostrem redução da mortalidade por câncer de mama por meio do rastreamento mamográfico em massa, ele também é alvo de controvérsias quanto a sua efetividade, sobretudo em mulheres abaixo dos 50 anos. Apesar disso, o rastreamento mamográfico em massa tem sido estimulado e praticado em mulheres a partir dos 40 anos, e apesar de suas limitações, ainda é o melhor método de rastreamento do câncer mamário disponível (Sclowitz, Menezes, Gigante, \& Tessaro, 2005).

O diagnóstico da neoplasia maligna da mama, comumente, é avassalador na vida da mulher que o recebe, seja pelo medo das mutilações e tratamentos agressivos, seja pelo medo de morrer ou pelas múltiplas perdas, no que tange aos aspectos social, econômico e psicológico, o que na maioria das vezes acontece (Oliveira, Policarpo, Silva, Silveira, \& Rodrigues, 2019).

Segundo Bergamasco e Angelo (2001) os profissionais de saúde também foram considerados elementos importantes no enfrentamento da doença, quando informavam sobre a doença e sua evolução, bem como quando encorajavam e confortavam a mulher.

Apesar do diagnóstico, na maioria das vezes, ainda ser feito em estágios mais avançados da doença, novos métodos para detecção precoce e novas possibilidades de tratamento vêm surgindo, resultando em um aumento da sobrevida dessas mulheres. Essa melhora da expectativa de vida se contrapõe a uma alteração no estado de saúde global, uma vez que o câncer da mama e os tratamentos propostos causam um grande impacto na vida dessas mulheres (Makluf, Dias, \& Barra, 2006).

Segundo Pereira, Gomes, \& Oliveira, (2017) o diagnóstico de câncer de mama e a possibilidade de mastectomia geram muitas incertezas, medos e ansiedades. Estudos apontam para uma "sequela psicológica", a qual pode ser mais grave que a própria deformidade deixada pela mastectomia. Portanto, sistematizar o conhecimento produzido acerca do cuidado destas mulheres pode contribuir para a instrumentalização de profissionais que atuam nessa área.

Após a seleção dos artigos e sua identificação, as publicações serão submetidas a um fichamento que será apresentado no Quadro 1.

Quadro 1 - Artigos selecionados para revisão, Salvador - BA.

\begin{tabular}{|c|c|c|c|c|}
\hline Ano & Autores & Título & $\begin{array}{ll}\text { Tipo } \\
\text { estudo }\end{array}$ & Principais achados \\
\hline 2001 & $\begin{array}{l}\text { Bergamasco, R. B., \& } \\
\text { Angelo, M. }\end{array}$ & $\begin{array}{l}\text { O sofrimento de descobrir-se } \\
\text { com câncer de mama: como } \\
\text { o diagnóstico é experienciado } \\
\text { pela mulher. }\end{array}$ & $\begin{array}{l}\text { Abordagem } \\
\text { qualitativa }\end{array}$ & $\begin{array}{l}\text { Compreensão da experiência da mulher no } \\
\text { enfrentamento do diagnóstico a partir dos elementos } \\
\text { componentes do seu sofrimento: surpresa, medo e } \\
\text { aprendizagem. }\end{array}$ \\
\hline 2003 & $\begin{array}{l}\text { Duarte, T. P., \& Andrade, } \\
\text { Â. N. de. }\end{array}$ & $\begin{array}{l}\text { Enfrentando a mastectomia: } \\
\text { análise dos relatos de } \\
\text { mulheres mastectomizadas } \\
\text { sobre questões ligadas à } \\
\text { sexualidade. }\end{array}$ & $\begin{array}{l}\text { Abordagem } \\
\text { qualitativa }\end{array}$ & $\begin{array}{l}\text { O diagnóstico do câncer de mama desencadeia uma } \\
\text { série de conflitos emocionais, a morte e a perda da } \\
\text { mama neste momento. É importante destacar que cada } \\
\text { mulher reage conforme algumas variáveis que dizem } \\
\text { respeito à sua história de vida, ao contexto social, } \\
\text { econômico e familiar de cada uma. }\end{array}$ \\
\hline 2003 & Thuler, L. C. & Considerações sobre a & Revisão da & A educação para promover o diagnóstico precoce e o \\
\hline
\end{tabular}




\begin{tabular}{|c|c|c|c|c|}
\hline & & $\begin{array}{l}\text { prevenção do câncer de } \\
\text { mama feminino. }\end{array}$ & $\begin{array}{l}\text { literatura não } \\
\text { sistemática. }\end{array}$ & $\begin{array}{l}\text { rastreamento. As evidências obtidas de ensaios } \\
\text { clínicos sugerem uma diminuição de } 25 \% \text { da } \\
\text { mortalidade por câncer de mama com o rastreamento } \\
\text { mamográfico de rotina. }\end{array}$ \\
\hline 2005 & \begin{tabular}{lrrr} 
Sclowitz, & \multicolumn{2}{c}{ M. } & L., \\
Menezes, & A. & M. & B., \\
Gigante, & D. & P., & \& \\
Tessaro, S. & & &
\end{tabular} & $\begin{array}{l}\text { Condutas na prevenção } \\
\text { secundária do câncer de } \\
\text { mama e fatores associados. }\end{array}$ & $\begin{array}{l}\text { Estudo } \\
\text { transversal. }\end{array}$ & $\begin{array}{l}\text { O câncer de mama é uma doença de extrema } \\
\text { importância para saúde pública em nível mundial. O } \\
\text { nível socioeconômico parece ser o principal } \\
\text { determinante do acesso à consulta ginecológica e, } \\
\text { consequentemente, às demais condutas na prevenção. }\end{array}$ \\
\hline 2006 & $\begin{array}{l}\text { Cantinelli, F. S., } \\
\text { Camacho, R. S., Smaletz, } \\
\text { O., Gonsales, B. K., } \\
\text { Braguittoni, É., \& Rennó, } \\
\text { J., Jr. }\end{array}$ & $\begin{array}{l}\text { A oncopsiquiatria no câncer } \\
\text { de mama: considerações a } \\
\text { respeito de questões do } \\
\text { feminino. }\end{array}$ & $\begin{array}{l}\text { Levantamento } \\
\text { Bibliográfico }\end{array}$ & $\begin{array}{l}\text { Possibilidades terapêuticas, imagem corporal e } \\
\text { impacto sobre auto-estima e sexualidade, tratamentos } \\
\text { sistêmicos e consequêencias físicas, tais como fadiga, } \\
\text { náuseas e vômitos, foram discutidos. Manter vigilância } \\
\text { quanto aos aspectos psíquicos de mulheres acometidas } \\
\text { com câncer de mama e à sua QV, em especial à } \\
\text { imagem corporal, saúde e desconfortos físicos, } \\
\text { trabalho e vida sexual. }\end{array}$ \\
\hline 2006 & $\begin{array}{l}\text { Pereira, S. G., Rosenhein, } \\
\text { D. P., Bulhosa, M. S., } \\
\text { Lunardi, V. L., \& Lunardi } \\
\text { Filho, W. D. }\end{array}$ & $\begin{array}{l}\text { Vivências de cuidados da } \\
\text { mulher mastectomizada: uma } \\
\text { pesquisa bibliográfica. }\end{array}$ & $\begin{array}{l}\text { Pesquisa } \\
\text { bibliográfica }\end{array}$ & $\begin{array}{l}\text { Sentimentos identificados nas mulheres: medo, } \\
\text { rejeição, culpa e perda. Como principal estratégia de } \\
\text { cuidado, foram identificadas as atividades em grupo, } \\
\text { visando promover a auto-estima da mulher } \\
\text { mastectomizada e contribuir para sua qualidade de } \\
\text { vida. }\end{array}$ \\
\hline 2008 & $\begin{array}{l}\text { Bertolo, B. L., \& Pauli, L. } \\
\text { T. S. }\end{array}$ & $\begin{array}{l}\text { O papel da enfermagem } \\
\text { como cuidadora nas questões } \\
\text { das fragilidades da mulher } \\
\text { pós-mastectomia. }\end{array}$ & $\begin{array}{l}\text { Caráter } \\
\text { exploratório e } \\
\text { descritivo, com } \\
\text { análise } \\
\text { qualitativa dos } \\
\text { dados. }\end{array}$ & $\begin{array}{l}\text { Para as mulheres, a ausência do seio provocou } \\
\text { sintomas de estranheza; entretanto, mesmo sendo uma } \\
\text { situação difícil e delicada, tiveram uma boa } \\
\text { aceitabilidade, pois a vida era considerada mais } \\
\text { importante do que a perda do órgão propriamente dito. } \\
\text { Demonstra o quanto os profissionais de saúde devem } \\
\text { intervir de forma qualitativa, realizando atividades de } \\
\text { prevenção e reabilitação da saúde da mulher. }\end{array}$ \\
\hline 2008 & Silva, L. C. da. & $\begin{array}{l}\text { Câncer de mama e } \\
\text { sofrimento } \\
\text { aspectos relacionados ao } \\
\text { feminino. }\end{array}$ & $\begin{array}{l}\text { Levantamento } \\
\text { Bibliográfico }\end{array}$ & $\begin{array}{l}\text { Contribui para que os profissionais de saúde, } \\
\text { principalmente os de saúde mental, possam assisti-la } \\
\text { de maneira mais eficiente e abrangente. O sofrimento } \\
\text { psicológico da mulher portadora de um câncer de } \\
\text { mama e de ter de acolher um tratamento difícil } \\
\text { transcende ao sofrimento configurado pela doença em } \\
\text { si. }\end{array}$ \\
\hline 2010 & $\begin{array}{l}\text { Moura, F. M. de J. S. de } \\
\text { P., Silva, M. G. da, } \\
\text { Oliveira, S. C. de, \& } \\
\text { Moura, L. de J. S. P. de. }\end{array}$ & $\begin{array}{l}\text { Os sentimentos das mulheres } \\
\text { pós-mastectomizadas. }\end{array}$ & $\begin{array}{l}\text { Pesquisa } \\
\text { Qualitativa }\end{array}$ & $\begin{array}{l}\text { Sentimentos gerados pela falta de apoio e atenção dos } \\
\text { profissionais durante a assistência. As dificuldades são } \\
\text { incalculáveis, requerem adaptações profundas no } \\
\text { modo de pensar e agir, que influencia física e } \\
\text { emocionalmente o convívio pessoal e social. }\end{array}$ \\
\hline 2012 & $\begin{array}{l}\text { Kluthcovsky, A. C. G. C., } \\
\text { \& Urbanetz, A. A. L. }\end{array}$ & $\begin{array}{l}\text { Qualidade de vida em } \\
\text { pacientes sobreviventes de } \\
\text { câncer de mama comparada à } \\
\text { de mulheres saudáveis. }\end{array}$ & $\begin{array}{l}\text { Estudo } \\
\text { transversal }\end{array}$ & $\begin{array}{l}\text { Os pontos fortes deste estudo foram o foco sobre a } \\
\text { qualidade de vida em sobreviventes de câncer de } \\
\text { mama e a inclusão de um Grupo Controle pareado por } \\
\text { idade. Muitas sobreviventes de câncer de mama } \\
\text { experimentaram piores avaliações na qualidade de } \\
\text { vida quando comparadas às mulheres saudáveis. }\end{array}$ \\
\hline 2013 & $\begin{array}{l}\text { Lopes, M. H. B. de M., } \\
\text { Moura, A. A. de, Raso, } \\
\text { S., Vedovato, T. G., \& } \\
\text { Ribeiro, M. A. S. }\end{array}$ & $\begin{array}{l}\text { Diagnósticos de enfermagem } \\
\text { no pós-operatório de } \\
\text { mastectomia. }\end{array}$ & $\begin{array}{l}\text { Estudo } \\
\text { descritivo e } \\
\text { retrospectivo }\end{array}$ & $\begin{array}{l}\text { Qualquer enfermeiro ao formular um diagnóstico } \\
\text { depara-se com a difícil tarefa de julgar. Diagnósticos } \\
\text { que exigem uma abordagem psicossocial para serem } \\
\text { identificados como Distúrbio na Imagem Corporal e } \\
\text { Angústia Espiritual. }\end{array}$ \\
\hline 2014 & $\begin{array}{l}\text { Ribeiro, I. F. A., Sousa, } \\
\text { R. D. F., Andrade, S. P., } \\
\text { Brito, M. C. C., } \\
\text { Albuquerque, I. M. N. }\end{array}$ & $\begin{array}{l}\text { Grupo de autoajuda com } \\
\text { mulheres mastectomizadas: } \\
\text { trabalhando estratégias de } \\
\text { educação em saúde. }\end{array}$ & $\begin{array}{l}\text { Exploratório- } \\
\text { descritivo, } \\
\text { natureza } \\
\text { qualitativa, } \\
\text { modalidade } \\
\text { pesquisa-ação. }\end{array}$ & $\begin{array}{l}\text { Relato de preconceito no ambiente de trabalho. Sinais } \\
\text { de edema, dormência e dificuldades de movimentação } \\
\text { em membro superior homolateral à mastectomia foram } \\
\text { citados, gerando sentimento de impotência. Partilhar } \\
\text { experiências vividas, uma vez que cada mulher reage a } \\
\text { situações conforme algumas variáveis respeitando a } \\
\text { sua história de vida, contexto social, econômico e } \\
\text { familiar. }\end{array}$ \\
\hline 2015 & $\begin{array}{l}\text { Lago, E. D. A., Andrade, } \\
\text { N. K. S., Nery, I. S., \& } \\
\text { Avelino, F. V. S. D. }\end{array}$ & $\begin{array}{l}\text { Sentimento de mulheres } \\
\text { mastectomizadas acerca da } \\
\text { autoimagem e alterações na } \\
\text { vida diária. }\end{array}$ & $\begin{array}{l}\text { Pesquisa } \\
\text { qualitativa }\end{array}$ & $\begin{array}{l}\text { Referiram ainda o abandono ou a redução de suas } \\
\text { atividades domésticas ou laborais, impostas pelas } \\
\text { limitações da mastectomia. A mastectomia gera um } \\
\text { misto de sentimentos e altera a imagem corporal, } \\
\text { autoestima, relações sociais e cotidianas. }\end{array}$ \\
\hline
\end{tabular}




\begin{tabular}{|c|c|c|c|c|}
\hline 2016 & $\begin{array}{l}\text { Peiter, C., Caminha, M., } \\
\text { Lanzoni, G., \& Erdmann, } \\
\text { A. }\end{array}$ & $\begin{array}{l}\text { Managing nursing care } \\
\text { delivery to cancer patients in } \\
\text { a general hospital: a } \\
\text { Grounded Theory. }\end{array}$ & $\begin{array}{l}\text { This } \\
\text { qualitative } \\
\text { research }\end{array}$ & $\begin{array}{l}\text { To understand the meaning of the management of } \\
\text { nursing care delivery to cancer inpatients in a general } \\
\text { hospital. }\end{array}$ \\
\hline 2017 & $\begin{array}{l}\text { Almeida-Brasil, C. C., } \\
\text { Silveira, M. R., Silva, K. } \\
\text { R., Lima, M. G., Faria, C. } \\
\text { D. C. de M., Cardoso, C. } \\
\text { L., Menzel, H.-J. K., \& } \\
\text { Ceccato, M. das G. B. }\end{array}$ & $\begin{array}{l}\text { Qualidade de vida e } \\
\text { características associadas: } \\
\text { aplicação do WHOQOL- } \\
\text { BREF no contexto da } \\
\text { Atenção Primária à Saúde. }\end{array}$ & $\begin{array}{c}\text { Análise } \\
\text { descritiva }\end{array}$ & $\begin{array}{l}\text { A QV pode ser definida como "a percepção do } \\
\text { indivíduo de sua posição no contexto da cultura e } \\
\text { sistema de valores nos quais ele vive e em relação aos } \\
\text { seus objetivos, expectativas, padrões e preocupaçoses. }\end{array}$ \\
\hline 2017 & $\begin{array}{l}\text { Pereira, G., Gomes, A., \& } \\
\text { Oliveira, R. }\end{array}$ & $\begin{array}{l}\text { Impacto do tratamento do } \\
\text { câncer de mama na } \\
\text { autoimagem } \text { e nos } \\
\text { relacionamentos afetivos de } \\
\text { mulheres mastectomizadas. }\end{array}$ & $\begin{array}{l}\text { Transversal } \\
\text { parte de um } \\
\quad \text { estudo } \\
\text { prospectivo }\end{array}$ & $\begin{array}{l}\text { O tratamento do câncer de mama promove impacto } \\
\text { negativo na autoimagem corporal e nos } \\
\text { relacionamentos afetivo e sexual com o cônjuge, no } \\
\text { entanto, favorece os relacionamentos fraternais. } \\
\text { Estudos sobre qualidade de vida podem colaborar no } \\
\text { entendimento desses impactos negativos. }\end{array}$ \\
\hline 2017 & $\begin{array}{l}\text { Rodrigues, N. S., Orsini, } \\
\text { M. R. C. A., Machado, A. } \\
\begin{array}{ll}\text { A., Montiel, J. } & \text { M., } \\
\text { Bartholomeu, } & \text { D., } \\
\text { Tertuliano, I. W. } & \end{array}\end{array}$ & $\begin{array}{l}\text { Importância do } \\
\text { acompanhamento psicológico } \\
\text { em mulher mastectomizada: } \\
\text { artigo de revisão. }\end{array}$ & $\begin{array}{l}\text { Pesquisa } \\
\text { analítica, } \\
\text { também } \\
\text { conhecida } \\
\text { como } \\
\text { bibliografia }\end{array}$ & $\begin{array}{l}\text { Falta de comunicação das pacientes prejudicando suas } \\
\text { relações ou fazendo com que ela se isole, o } \\
\text { acompanhamento deve auxiliar essa paciente a romper } \\
\text { pouco a pouco esse silêncio, com o objetivo de evitar } \\
\text { que possíveis problemas decorrentes dessa falta de } \\
\text { comunicação surjam. A importância de se oferecer } \\
\text { suporte aos parceiros de mulheres mastectomizadas. }\end{array}$ \\
\hline 2018 & $\begin{array}{l}\text { Monteiro, C. R. A. V., \& } \\
\text { Paiva, A. R. B. }\end{array}$ & $\begin{array}{l}\text { Qualidade de vida em } \\
\text { mulheres mastectomizadas. }\end{array}$ & $\begin{array}{l}\text { Qualitativo, } \\
\text { descritivo, } \\
\text { observacional } \\
\text { e transversal }\end{array}$ & $\begin{array}{l}\text { Trauma psicológico, quanto ao tratamento e medo da } \\
\text { mutilação e distorção da autoimagem; A importância } \\
\text { da assistência multidisciplinar no acompanhamento } \\
\text { pós-operatório de mulheres mastectomizadas, com } \\
\text { ênfase na reabilitação. }\end{array}$ \\
\hline 2018 & $\begin{array}{l}\text { Silva, F. C., Ferreira, L. } \\
\text { J., Costa, C. M., \& } \\
\text { Pernambuco, A. P. }\end{array}$ & $\begin{array}{l}\text { Avaliação da qualidade de } \\
\text { vida em mulheres com } \\
\text { câncer de mama submetidas } \\
\text { à intervenção cirúrgica. }\end{array}$ & $\begin{array}{l}\text { Análise estatí- } \\
\text { stica descritiva }\end{array}$ & $\begin{array}{l}\text { Contudo, a maior sobrevida quase sempre é } \\
\text { acompanhada de comorbidades físicas e ou } \\
\text { psicológicas que podem impactar na qualidade de } \\
\text { vida destas mulheres. }\end{array}$ \\
\hline 2019 & $\begin{array}{l}\text { Cabral, A. L. L. V., } \\
\text { Giatti, L., Casale, C., \& } \\
\text { Cherchiglia, M. L. }\end{array}$ & $\begin{array}{l}\text { Vulnerabilidade social e } \\
\text { câncer de mama: diferenciais } \\
\text { no intervalo entre o } \\
\text { diagnóstico e o tratamento } \\
\text { em mulheres de diferentes } \\
\text { perfis sociodemográficos. }\end{array}$ & $\begin{array}{l}\text { Estudo } \\
\text { transversal }\end{array}$ & $\begin{array}{l}\text { Observou-se que o estadiamento do câncer pouco } \\
\text { influenciou na determinação do intervalo entre o } \\
\text { diagnóstico e tratamento. Com prejuízo para as } \\
\text { mulheres com características sociais de maior } \\
\text { vulnerabilidade, que prevalecem mesmo após vencidas } \\
\text { as barreiras de acesso à unidade de tratamento } \\
\text { oncológico. }\end{array}$ \\
\hline 2019 & $\begin{array}{l}\text { Oliveira, P. P., Policarpo, } \\
\text { A. G., Silva, L. M. da, } \\
\text { Silveira, E. A. A. da, \& } \\
\text { Rodrigues, A. B. }\end{array}$ & $\begin{array}{l}\text { Vivências de mulheres com } \\
\text { câncer de mama: uma } \\
\text { pesquisa-cuidado. }\end{array}$ & $\begin{array}{l}\text { Estudo } \\
\text { qualitativo }\end{array}$ & $\begin{array}{l}\text { Efetivou-se um cuidado humanizado e educativo, com } \\
\text { orientações e educação em saúde, possibilitando } \\
\text { suporte ao processo saúde - adoecimento-tratamento } \\
\text { das pesquisadas-cuidadas. Promoveram-se ações } \\
\text { fundamentadas não só no conhecimento científico, } \\
\text { mas no cuidado transpessoal com interação e troca } \\
\text { entre o ser-pesquisado e o ser-pesquisador. }\end{array}$ \\
\hline 2019 & $\begin{array}{l}\text { Reis, A. } \quad \text { P. } \\
\text { Panobianco, M. } \\
\text { Gradim, C. V. C. }\end{array}$ & $\begin{array}{l}\text { Enfrentamento de mulheres } \\
\text { que vivenciaram o câncer de } \\
\text { mama. }\end{array}$ & $\begin{array}{l}\text { Estudo } \\
\text { qualitativo }\end{array}$ & $\begin{array}{l}\text { Demostra que a mulher que vivencia o câncer de } \\
\text { mama utiliza-se de aspectos de resiliência para } \\
\text { enfrentar a sociedade, da família para oferecer suporte } \\
\text { e de aspectos da experiência de vida. Este estudo } \\
\text { contribui para impulsionar mudanças, transformações } \\
\text { e inovações, tanto em nível pessoal, como profissional } \\
\text { e institucional na assistência a essas mulheres. }\end{array}$ \\
\hline
\end{tabular}

Fonte: Autores (2021).

Mediante leitura e fichamento dos artigos, foras identificadas duas categorias temáticas: Realizar uma revisão de literatura sobre o cuidado de enfermagem as mulheres após a cirurgia de mama: mastectomia e identificar os cuidados de enfermagem e a qualidade de vida à mulher mastectomizada. 


\section{Cirurgia de Mama: Mastectomia}

A retirada da mama está, geralmente, acompanhada de consequências traumatizantes nas experiências de vida de uma mulher, visto o desencadeamento de uma série de distúrbios que ocorrem. Essas dificuldades e desajustes sociais terminam por desencadear sentimentos negativos, como o choque emocional causado pelo diagnóstico; o medo da cirurgia; a incerteza do prognóstico e de uma recorrência deste câncer; os efeitos da rádio e da quimioterapia; o medo da dor e o pavor de encarar a morte nas circunstâncias que, em geral, acontece (Ribeiro, Sousa, Andrade, Brito, \& Albuquerque, 2014).

As atividades terapêuticas são planejadas de acordo com esses estágios. É imprescindível o conhecimento do estágio clínico da paciente, visto que este permite estabelecer a extensão da área atingida e a gravidade da doença, além de planejar um tratamento adequado, assim como o prognóstico. A escolha do método terapêutico apropriado irá depender, também, de vários fatores, tais como a idade da paciente, a localização e o tamanho do tumor, a disponibilidade financeira, a análise da mamografia e o modo da paciente lidar com a mama afetada (Duarte \& Andrade, 2003).

Segundo Silva (2018) o tratamento primário é a mastectomia, intervenção cirúrgica que pode ser restrita ao tumor, atingir tecidos circundantes ou até a retirada da mama, dos linfonodos da região axilar e de ambos os músculos peitorais. A mais frequente, em torno de $57 \%$ das intervenções realizadas, é a mastectomia radical modificada, aquela que remove toda a mama juntamente com os linfonodos axilares. Tratamentos complementares geralmente são necessários, como a radioterapia, quimioterapia e hormonioterapia. O prognóstico e a escolha do tratamento são embasados na idade da paciente, estágio da doença, características do tumor primário, níveis de receptores de estrógeno e de progesterona, medidas de capacidade proliferativa do tumor, situação da menopausa e saúde geral da mulher.

Segundo Moura et al. (2010) o tratamento mais comum é a extração da mama comprometida. Em alguns casos, os médicos vêm fazendo apenas a retirada de partes da mama, através de: quadrantectomia (remoção de um quarto da mama) e lumpectomia (remoção apenas do tumor e de pequena região circunvizinha), obtendo assim bons resultados em termos de sobrevida e melhor efeito estético, já que o órgão é conservado.

A mastectomia parcial ou radical modificada, dentre outros tratamentos existentes, é a mais indicada para o câncer de mama. Esta modalidade sofreu grandes avanços nos últimos tempos devido à evolução científica, e a técnica cirúrgica de mastectomia com reconstrução mamária imediata pode ser considerada um exemplo deste avanço (Lopes et al., 2013).

A mastectomia é um dos tratamentos prováveis para a maioria das mulheres com câncer de mama. Ao submeter-se à retirada da mama ou parte dela, certamente, a mulher estará passando por uma grande mudança, vivenciando, assim, um comprometimento físico, emocional e social. A cirurgia e sua associação a outros tratamentos para o câncer podem interromper os hábitos de vida da mulher, provocando alterações nas suas relações familiares e sociais, quase sempre provenientes, também, de sentimento de impotência e de frustração sobre algo que foge ao seu controle, como o próprio temor da doença (Pereira et al., 2006).

Segundo Duarte e Andrade (2003) a reconstrução da mama nesse âmbito representa uma possibilidade de reabilitação bastante atual para as mulheres que necessitam realizar a mastectomia radical como o tratamento principal para o câncer de mama. Essa reconstrução dependerá de vários aspectos: peso, altura, idade, tratamento prévio ou complementar com radioterapia, estado de saúde e outras cirurgias que a paciente já tenha realizado. O avanço das técnicas de cirurgia plástica nos últimos anos tem proporcionado resultados satisfatórios para a expectativa estética e psicológica da mulher, ao reduzir o trauma causado pela mutilação.

A reconstrução da mama é uma alternativa para reabilitação e eufêmica o impacto psicológico sofrido pelas mulheres mastectomizadas. Nessa conjuntura, a enfermagem deve compreender os sentimentos das mulheres mastectomizadas, a fim de diagnosticar as principais necessidades e realizar um planejamento de enfermagem mais coerente na assistência a essas (Lago, Andrade, Nery, \& Avelino, 2015). 
É bom lembrar que a mama não corresponde apenas ao aspecto físico, ela é vista como um símbolo de feminilidade, por isso é motivo de orgulho para a mulher e admiração para os homens, possuindo um significado afetivo e psicológico. Sendo assim, a palavra câncer traz um estigma muito forte para a mulher, pois além de estar associada à morte, pode afetar uma parte tão valorizada do seu corpo (Moura et al., 2010).

Segundo Cantinelli et al. (2010) uma das complicações da cirurgia é o desenvolvimento de linfedema no membro superior após a dissecção de linfonodos axilares. Além de a paciente sentir-se mutilada pela cirurgia, o linfedema causa alterações importantes não só físicas como funcionais. Recomenda-se que, após a dissecção de linfonodos axilares, não sejam feitos movimentos bruscos com o membro superior, se evitem infecções nesse membro, não se permita a colocação de agulhas etc. Recentemente, a técnica do linfonodo sentinela possibilitou a diminuição do número de pacientes com essa complicação.

As repercussões psicológicas provocadas pela cirurgia do câncer de mama, por si só, justificam a importância de se oferecer suporte a mulheres mastectomizadas. O psicólogo aparece nesse cenário como figura de apoio para que o sofrimento psíquico experimentado pela paciente possa ser expresso. De modo geral, a ideia é permitir que a mulher vivencie não apenas cada uma das fases do tratamento, mas também cada reação emocional que é suscitada por essas diversas etapas (Rodrigues et al., 2017).

\section{Qualidade de Vida e a Enfermagem}

Qualidade de vida é definida pela Organização Mundial da Saúde (OMS) como: “[...] a percepção do indivíduo de sua posição na vida no contexto da cultura e sistema de valores nos quais ele vive e em relação aos seus objetivos, expectativas, padrões e preocupações". Esta definição da OMS deixa implícita a ideia de que o conceito de qualidade de vida é subjetivo, multidimensional e inclui elementos de avaliação tanto positivos como negativos (Kluthcovsky \& Urbanetz, 2012).

Segundo Makluf, Dias e Barra (2006) a qualidade de vida inclui uma variedade potencial maior de condições que podem afetar a percepção do indivíduo, seus sentimentos e comportamentos relacionados com o seu funcionamento diário, incluindo, mas não se limitando, à sua condição de saúde e às intervenções médicas, e se apoia na compreensão das necessidades humanas fundamentais, materiais e espirituais, e tem no conceito de promoção de saúde seu foco mais relevante. Relaciona-se à saúde (QVRS) e estado subjetivo de saúde são conceitos afins, centrados na avaliação subjetiva do paciente, mais necessariamente ligados ao impacto do estado de saúde sobre a capacidade do indivíduo viver plenamente.

As mulheres que tiveram perdas mamárias, por vezes, deixam de ter um relacionamento social. Suas atividades de lazer já não são mais as mesmas, sua carreira é comprometida, como também sua vida familiar. Depois de tantas mudanças em sua vida, começam suas preocupações relacionadas ao próprio corpo. Nesse momento, geralmente se sentem satisfeitas com o término do tratamento, mas se encontram mentalmente e fisicamente esgotadas pelos procedimentos invasivos e dolorosos (Pereira, Gomes, \& Oliveira, 2017).

Uma das funções do enfermeiro é assegurar a qualidade de vida da mulher mastectomizada por meio de ações educativas, sendo tão relevantes quanto os cuidados clínicos. Assim, a experiência da mastectomia pode ser menos traumática para as mulheres com câncer de mama se houver pleno empenho dos enfermeiros ao considerarem os aspectos psicossociais16, a fim de estabelecerem diagnósticos de enfermagem que embasam essas ações (Lopes et al., 2013).

Segundo Peiter, Caminha, Lanzoni e Erdmann (2016) neste sentido, a gestão do cuidado de enfermagem é uma ferramenta importante para a qualificação da assistência, uma vez que procura o cuidado integral, observando as perspectivas necessárias para alcançar um cuidado individualizado. Para isso, faz uso de instrumentos como o domínio sobre a organização dos serviços de saúde, a sua interface com os serviços de apoio e a sistematização da assistência de enfermagem. 
A equipe de enfermagem e a equipe multidisciplinar precisam voltar-se para aspectos de humanização do atendimento, cuidados com a qualidade de vida das pacientes após a mastectomia, uma vez que é fundamental para a transformação dos processos de evolução do auto cuidado e da aceitação da nova fase. Os aspectos emocionais da paciente e da família devem ser vistos de forma especial, partindo do ponto de quem a unidade de oncológica é um ambiente estressante e desgastante no qual o apoio da equipe de enfermagem pode ser essencial para amenizar os conflitos existentes.

Segundo Pereira, Gomes e Oliveira (2017) essa enfermidade também produz alterações importantes no corpo e na autoimagem da mulher, que podem afetar na parte de sua sexualidade e sua satisfação conjugal. Tais interferências são normalmente pela experiência de sentir seu corpo em mudança, perda do cabelo, perda da mama e ressecamento vaginal, que leva a dor e desconforto na hora do ato sexual. Aos poucos, e após a fase aguda do tratamento, há uma tendência a se retornar à qualidade de vida prévia, e também as muitas mudanças positivas, com oportunidades de crescimento pessoal. O enfrentamento da doença leva, na maioria dos casos, a um maior sentido social, pessoal e até mesmo profissional.

Podendo a equipe de enfermagem desenvolver um atendimento qualificado e sistemático através de uma qualificação eficaz, voltada por meio de um acompanhamento multidisciplinar, com protocolos de atendimento diferenciado, com objetivos de diminuir o impacto da nova fase da mulher no pós cirúrgico, de proporcionar uma assistência voltada também aos anseios com relação ao momento que está vivendo e com ênfase no conflito com a sexualidade.

A equipe de enfermagem está voltada sempre para as questões de educação, orientação e cuidado para com a paciente, e dessa forma a mesma deve estar atenta para o atendimento qualificado e humanizado para com as mulheres, para que haja a diminuição da sensação de insegurança relatada pelas mesmas, na hora dos procedimentos pertinentes a unidade, nos cuidados que devem realizar em casa e em relação aos aspectos psicológicos.

A equipe deve estar sempre preparada para orientar e acolher a paciente e sua família da melhor forma possível, por intermédio de um embasamento teórico aprofundado nos problemas para que possa amenizar o sofrimento dessas mulheres.

\section{Conclusão}

Ao iniciar este estudo, pretendíamos abordar a importância do atendimento eficaz de enfermagem na oncologia, especificamente no acolhimento as pacientes após a cirurgia das mamas, e compreender as expectativas da paciente e tentar sanar alguns anseios da mesma junto ao acompanhante tentando colocar o cuidar da equipe de enfermagem em prol do âmbito oncológico e psicológico.

Ao analisar estudos já publicados, podemos desvelar que a unidade oncológica oferece serviços de alta complexidade e diversidade no atendimento a pacientes em situação de diagnostico, tratamento. Na qual as pacientes sentem-se fragilizadas, abatidas e devastadas emocionalmente com a nova fase a ser enfrentada, é nesse momento que precisam não só da enfermagem como da equipe multiprofissional.

No entanto, é relevante observar o quanto esses atendimentos humanizados e em equipe pode ajudar as pacientes a superar essa fase e conseguir se inserir novamente na sociedade e conquistar seu papel como esposa e mãe. Esse suporte que a equipe proporciona pode garantir uma qualidade da assistência e do tratamento.

Entretanto foi possível observar que é de fundamental importância que todos os envolvidos no cuidar estejam preparados, para desenvolver habilidades nos momentos de conflitos ou de emergências presenciada em unidade oncológica, considerando-se que é necessário que exista a humanização do cuidado neste processo vivenciado na unidade de acolhimento as pacientes após a realização da cirurgia das mamas, evidenciando a dificuldade do momento apresentado a essas mulheres e ao acompanhante, ou parceiro e no todo família. 
Por fim podemos observar o tão quanto é importante o gerenciamento do cuidado humanizado e eficaz para obter êxito no atendimento de qualidade, o quanto é fundamental o desempenho profissional da equipe que abrangem toda uma rede multidisciplinar oncológica para o resgate da qualidade de vida dessas pacientes no processo de adequação a essa nova fase.

É relevante pontuar a fundamental importância de novos estudos na abordagem do tema, frente a grande necessidade do amplo impacto da mastectomia remete as pacientes, faz junção aos cuidados devidos e humanizados a dinâmica de acolhimento sempre está voltado aos estudos relevantes e crescentes na área, é preciso um embasamento teórico com referencias precisas.

\section{Referências}

Almeida-Brasil, C. C., Silveira, M. R., Silva, K. R., Lima, M. G., Faria, C. D. C. de M., Cardoso, C. L., Menzel, H.-J. K., \& Ceccato, M. das G. B. (2017). Qualidade de vida e características associadas: aplicação do WHOQOL-BREF no contexto da Atenção Primária à Saúde. Ciencia \& saude coletiva, 22(5), 1705-1716.

Bergamasco, R. B., \& Angelo, M. (2001). O sofrimento de descobrir-se com câncer de mama: como o diagnóstico é experienciado pela mulher. Revista Brasileira de Cancerologia, 47(3), 277-282.

Bertolo, B. L., \& Pauli, L. T. S. (2008). O papel da enfermagem como cuidadora nas questões das fragilidades da mulher pós-mastectomia. Boletim da Saúde, 22(1).

Cabral, A. L. L. V., Giatti, L., Casale, C., \& Cherchiglia, M. L. (2019). Vulnerabilidade social e câncer de mama: diferenciais no intervalo entre o diagnóstico e o tratamento em mulheres de diferentes perfis sociodemográficos. Ciencia \& saude coletiva, 24(2), 613-622.

Cantinelli, F. S., Camacho, R. S., Smaletz, O., Gonsales, B. K., Braguittoni, É., \& Rennó, J., Jr. (2006). A oncopsiquiatria no câncer de mama: considerações a respeito de questões do feminino. Revista de Psiquiatria Clinica, 33(3), 124-133.

Duarte, T. P., \& Andrade, Â. N. de. (2003). Enfrentando a mastectomia: análise dos relatos de mulheres mastectomizadas sobre questões ligadas à sexualidade. Estudos de Psicologia, 8(1), 155-163.

Gil, A. C. (1994). Como elaborar projetos de pesquisa (3a ed.). Atlas.

Gil, A. C. (2002). Como elaborar projetos de pesquisa (4a ed.). Atlas.

Gomes, I. S., \& Caminha, I. D. O. (2013). Guia para estudos de revisão sistemática: uma opção metodológica para as ciências do movimento humano. Movimento (ESEFID/UFRGS), 20(1), 395.

Kluthcovsky, A. C. G. C., \& Urbanetz, A. A. L. (2012). Qualidade de vida em pacientes sobreviventes de câncer de mama comparada à de mulheres saudáveis. Revista Brasileira de Ginecologia e Obstetricia: Revista Da Federacao Brasileira Das Sociedades de Ginecologia e Obstetricia, 34(10), 453-458.

Lago, E. D. A., Andrade, N. K. S., Nery, I. S., \& Avelino, F. V. S. D. (2015). Sentimento de mulheres mastectomizadas acerca da autoimagem e alterações na vida diária. Ciência \& Saúde, 8(1), 15.

Lopes, M. H. B. de M., Moura, A. A. de, Raso, S., Vedovato, T. G., \& Ribeiro, M. A. S. (2013). Diagnósticos de enfermagem no pós-operatório de mastectomia. Escola Anna Nery, 17(2), 354-360.

Monteiro, C. R. A. V., \& Paiva, A. R. B. (2018). Qualidade de vida em mulheres mastectomizadas. Revista de Investigação Biomédica, 10(1), 30.

Moura, F. M. de J. S. de P., Silva, M. G. da, Oliveira, S. C. de, \& Moura, L. de J. S. P. de. (2010). Os sentimentos das mulheres pós-mastectomizadas. Escola Anna Nery, 14(3), 477-484.

Oliveira, P. P., Policarpo, A. G., Silva, L. M. da, Silveira, E. A. A. da, \& Rodrigues, A. B. (2019). Vivências de mulheres com câncer de mama: uma pesquisacuidado. Online Brazilian Journal of Nursing, 17(1), 8.

Peiter, C., Caminha, M., Lanzoni, G., \& Erdmann, A. (2016). Managing nursing care delivery to cancer patients in a general hospital: a Grounded Theory. Revista de Enfermagem Referência, IV Série(11), 61-69.

Pereira, G., Gomes, A., \& Oliveira, R. (2017). Impacto do tratamento do câncer de mama na autoimagem e nos relacionamentos afetivos de mulheres mastectomizadas. Life Style, 4(1), 99-118.

Pereira, S. G., Rosenhein, D. P., Bulhosa, M. S., Lunardi, V. L., \& Lunardi Filho, W. D. (2006). Vivências de cuidados da mulher mastectomizada: uma pesquisa bibliográfica. Revista Brasileira de Enfermagem, 59(6), 791-795.

Reis, A. P. A., Panobianco, M. S., \& Gradim, C. V. C. (2019). Enfrentamento de mulheres que vivenciaram o câncer de mama. Revista de Enfermagem Do Centro-Oeste Mineiro, 9. https://doi.org/10.19175/recom.v9i0.2758.

Ribeiro, I. F. A., Sousa, R. D. F., Andrade, S. P., Brito, M. C. C., \& Albuquerque, I. M. N. (2014). Grupo de autoajuda com mulheres mastectomizadas: trabalhando estratégias de educação em saúde. Sanare, 3(1), 35-40. 
Research, Society and Development, v. 10, n. 13, e183101321087, 2021

(CC BY 4.0) | ISSN 2525-3409 | DOI: http://dx.doi.org/10.33448/rsd-v10i13.21087

Rodrigues, N. S., Orsini, M. R. C. A., Machado, A. A., Montiel, J. M., Bartholomeu, D., \& Tertuliano, I. W. (2017). Importância do acompanhamento psicológico em mulher mastectomizada: artigo de revisão. Arq. Catarin Med, 46(1), 164-172.

Sclowitz, M. L., Menezes, A. M. B., Gigante, D. P., \& Tessaro, S. (2005). Condutas na prevenção secundária do câncer de mama e fatores associados. Revista de Saude Publica, 39(3), 340-349.

Selltz, C., Jahoda, M., Deutsch, M., Cook, S. (1967). Métodos de pesquisa nas relações sociais. São Paulo: Ed. Herder.

Silva, F. C., Ferreira, L. J., Costa, C. M., \& Pernambuco, A. P. (2018). Avaliação da qualidade de vida em mulheres com câncer de mama submetidas à intervenção cirúrgica. Fisioterapia Brasil, 19(4), 524-531.

Silva, L. C. da. (2008). Câncer de mama e sofrimento psicológico: aspectos relacionados ao feminino. Psicologia Em Estudo, 13(2), $231-237$.

Thuler, L. C. (2003). Considerações sobre a prevenção do câncer de mama feminino. Revista Brasileira de Cancerologia, 49(4), $227-238$. 